\title{
Early Vision and Image Processing: Evidences Favouring a Dynamic Receptive Field Model
}

\author{
Kuntal Ghosh ${ }^{1}$, Sandip Sarkar ${ }^{1}$, and Kamales Bhaumik ${ }^{2}$ \\ ${ }^{1}$ Saha Institute of Nuclear Physics, 1/AF Bidhannagar, Kolkata-64, India \\ kuntal.ghosh@saha.ac.in \\ ${ }^{2}$ West Bengal University of Technology, BF-142 Bidhannagar, Kolkata-64, India \\ kamales_bhaumik@yahoo.com
}

\begin{abstract}
Evidences favouring a dynamic receptive field model of retinal ganglion cells and the cells of Lateral Geniculate Nucleus (LGN) have been presented based on the perception of some brightness-contrast illusions. Of the different kinds of such stimuli, four, namely the Simultaneous Brightness-contrast, the White effect, the DeValois and DeValois checkerboard illusion and the Howe stimulus have been chosen to establish this model. The present approach attempts to carry forward the works that look upon visual perception as a step-by-step information processing task rather than a rule-based Gestalt approach and provides a new biologically inspired tool for simultaneous smoothing and edge enhancement in image processing.
\end{abstract}

\section{Introduction}

The present work is aimed at understanding and explaining some of the aspects of visual signal processing mainly at the retinal level and in the simple cells of primary visual cortex. The topic may be initiated by recalling a memorable observation of J. B. Barlow [1] :

A description of that activity of a single nerve cell which is transformed to and influences other nerve cells and of a nerve cell's response to such influences from other cells, is a complete enough description for functional understanding of the nervous system. There is nothing else "looking at" or controlling this activity, which must therefore provide a basis for understanding how the brain controls behaviour.

Assumption of such a position was no doubt a great leap forward in unfurling the "mysteries" of the functioning of the nervous system, probably the first straightforward physical and materialistic approach to achieve an understanding of it in contrast to the Gestalt or holistic approach. Yet in spite of being essentially objective in its spirit, it could not but finally surrender itself amidst the relics of anti-science subjectivism. This resulted from its mechanical mode of relying upon the attempts to provide perceptual explanations by means of single cell recordings only. The "part" alone thus assumed importance and the "whole" lost its legitimate role. It was forgotten that the part and the whole are only 
interrelated concepts and truth always emerges from a dialectical synthesis of the two. The success of Hubel-Wiesel's work [23], wherein they could model the activity of a deep-seated neuron of layer VI as an output of a summation circuit having inputs from neurons of outer plexiform layer, led to an over-emphasis in the study of the details of the neuronal circuits in the understanding of the functions of brain, completely ignoring the role of emergent behaviours of complex systems. A faith was spread that for any conceivable complicated human perception, one would be able to identify a type of neurons in the brain leading to the final output of the perception and the study of the circuits connected with that cell would lead to a complete understanding of the mechanism of that perception. What therefore started as a beginning of a new direction in neuroscience that was trying to rid itself of its mystical cloaks, led to unnecessary new mysticisms through the so-called discoveries like the grandmother detecting cell. Yet the basic point on which this approach initiated by Barlow [1] tried to emphasize, was that it is only neurons and neurons alone that are responsible for the entire information processing in nervous system. Like, for example in vision, starting from the two dimensional intensity array formation on the retina to the three dimensional object reconstruction and recognition in higher regions of the brain, the entire process is controlled and executed by networks of neurons of different types. This pro-materialistic approach, ushered in the radical thought process that there is no "soul " sitting anywhere and interpreting things from the neuronal outputs, but rather it is a collective, step-by-step synchronization of the outputs at various stages in the eye and the brain, no matter how complex that process is, that ultimately creates a perception of the world around us. It was this approach that generated vigor and excitement among the science community which needs to be carried forward in the proper perspective by eliminating all remnants of subjectivism.

The most likely approach in this direction would be to initially consider primary visual processing and start with gray scale scenario only. To get an insight into this, the methodology that we are going to adopt in the present work, is to study the brightness perception in case of some select brightness-contrast illusions. We shall first consider the well-known low-level illusion namely the Simultaneous Brightness-contrast stimulus, which is easily explainable with the well acclaimed Difference of Gaussian (DoG) model of the receptive field of retinal ganglion cells and the cells of lateral geniculate nucleus (LGN) 4. Then we shall consider the more complex stimuli like the White effect [5], and the DeValois and DeValois checkerboard illusion [6] which are often thought to be inexplainable without a Gestalt approach [7. We shall explain these effects by extending the DoG model that serves to support a dynamic model of the receptive field based on a linear combination of three Gaussians. Finally we shall consider a very interesting variant of the White effect stimulus called the Howe stimulus [8], that itself challenges the Gestalt school explanation and apply the above dynamic model of the receptive field to provide a considerably satisfactory explanation to this phenomenon as well. Finally, as a corollary, we shall also see how this model may provide a new approach to edge enhancement in images. 


\section{The Proposed Model}

\subsection{The Classical Model}

It is well-known that the brightness of a region of visual space is not related to the luminance of that region alone, but also depend on the luminance of its adjacent regions. When the brightness of a test region shifts away from the brightness of the surround region the phenomenon is termed as a brightness-contrast event. Such events, like the Mach band, the Simultaneous Brightness-contrast, the Hermann grid illusion or the grating induction effect can be more or less explained with the help of the isotropic DoG model of primary visual processing 4, even though there are still many subtle aspects of these stimuli that require a deeper understanding of how even these low-level brightness-contrast illusions are actually processed 910. Let us first consider the Simultaneous Brightness-contrast (SBC) illusion shown in Figure 1. This is a well-studied textbook example where a gray patch on a white background looks darker than an equiluminant gray patch on a black background. This model is well-explained by the classical DoG model. If Figure 1 is convoluted with a DoG filter given by:

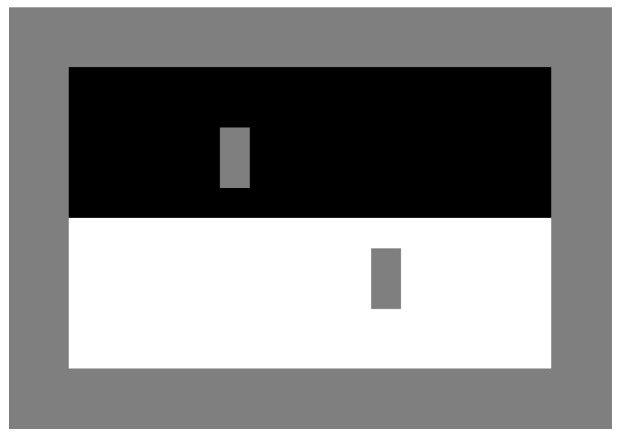

Fig. 1. The Simultaneous Brightness-Contrast illusion

$$
\operatorname{DOG}\left(\sigma_{1}, \sigma_{2}\right)=A_{1} \frac{1}{\sqrt{2 \pi} \sigma_{1}} \exp \left(-\frac{x^{2}}{2 \sigma_{1}^{2}}\right)-A_{2} \frac{1}{\sqrt{2 \pi} \sigma_{2}} \exp \left(-\frac{x^{2}}{2 \sigma_{2}^{2}}\right)
$$

then the convoluted image clearly bears the signature of our illusory perception. Such a convoluted image has been shown in Figure 2 (a). That the convoluted image is no illusion again has easily been confirmed by drawing two horizontal line profiles through the two test patches as shown in Figure 2(b). The result is easily understandable because the inhibition for the upper test patch from its dark surrounding makes it look brighter than that of the lower test patch with its bright surrounding. 
(a)

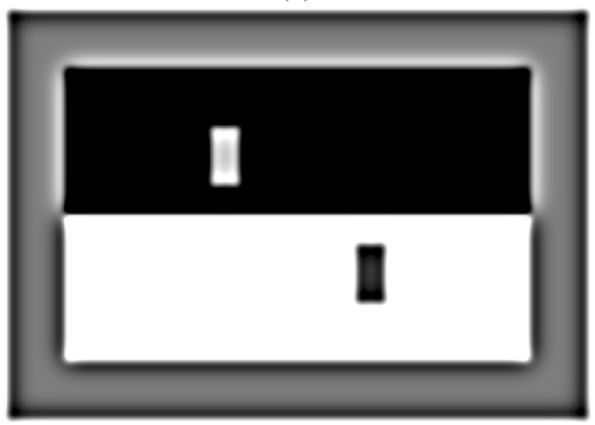

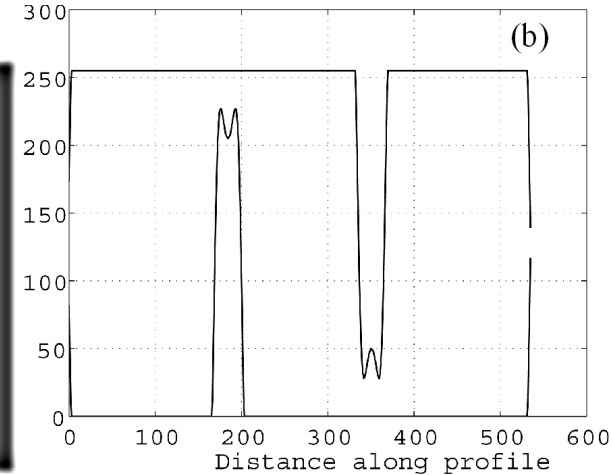

Fig. 2. (a) The Simultaneous Brightness-contrast stimulus when convoluted with DoG (b) Horizontal line profiles through the two test patches in (a). Clearly the test patch in brighter neighbourhood has become darker.

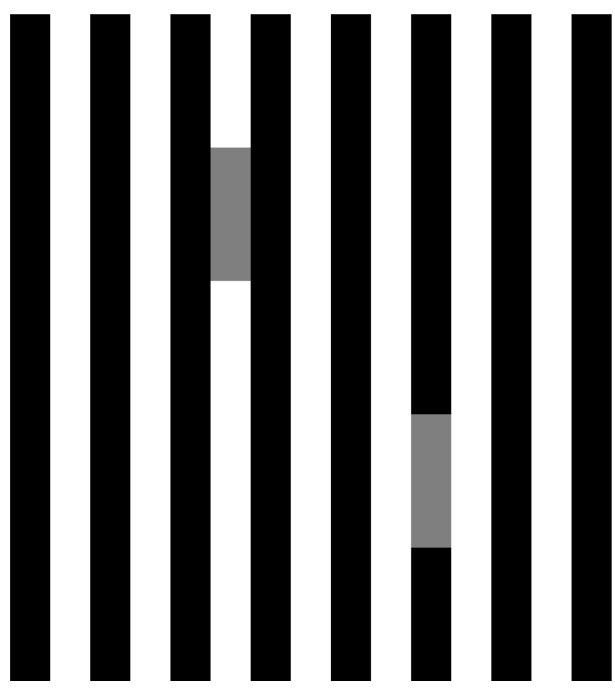

Fig. 3. The White Effect

\subsection{The Classical Model Modified}

We now consider the case of the White effect [5] which has caused much hype among the Gestalt theorists since it totally contradicts the lateral inhibition based theory of primary visual processing. Let us take a look at the White effect stimulus shown in Figure 3. In this effect, the direction of brightness change does not consistently correlate with the amount of black or white border in contact with the gray test patch or in its general vicinity. It can easily be seen from Figure 3 that the same vertically oriented rectangle that we used in the SBC 

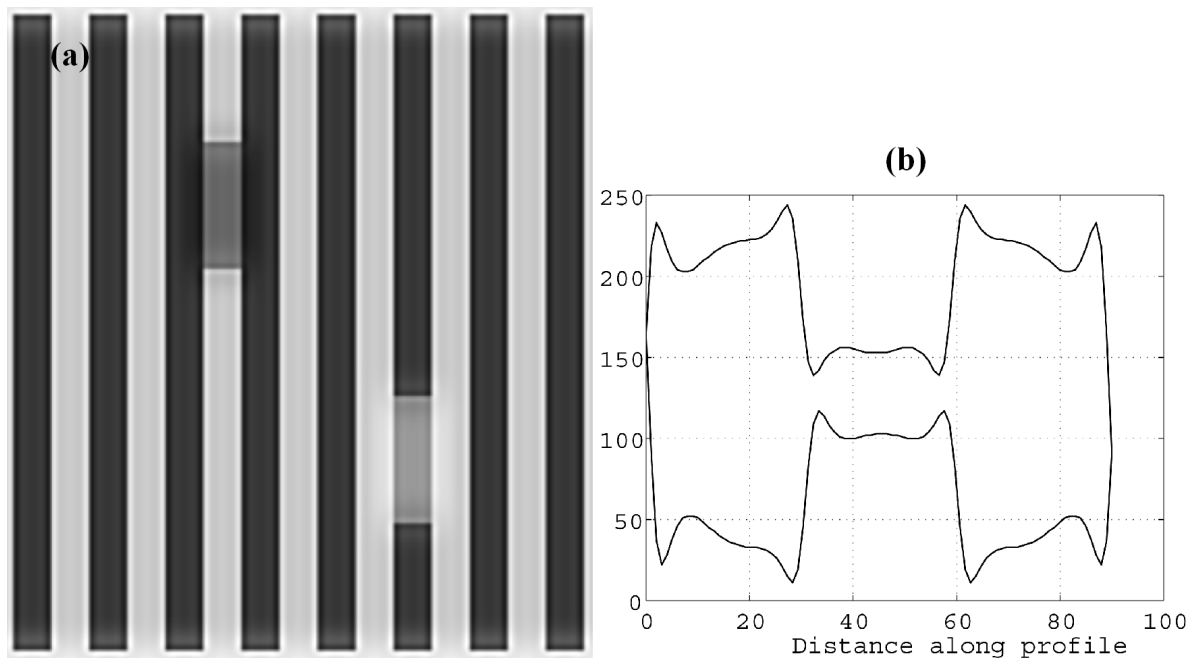

Fig. 4. (a) The White effect stimulus when convoluted with MDoG. (b) Horizontal line profiles through the two test patches in (a). Here unlike in Figure 2(b), the test patch in brighter neighbourhood has become brighter.

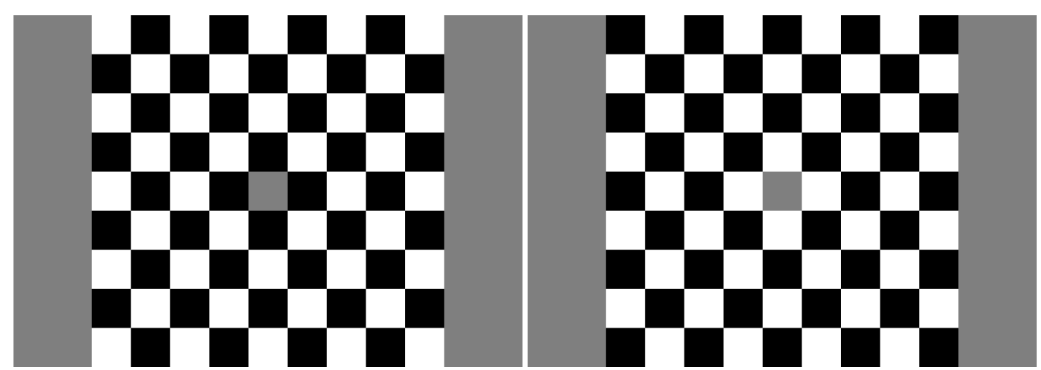

Fig. 5. The DeValois and DeValois Checkerboard stimulus

(Figure 1), when sitting on the white stripe of a vertical grating, appears darker than an identical test patch sitting on a black stripe. Because the later test patch has more border contact with the black flanking bars and yet appears darker, the effect is opposite to the SBC and hence cannot be explained by lateral inhibition. Most of the explanations of White effect provided so far are not computational models, except that of Ross and Pessoa [11. But the White effect can also be explained in the light of a simple modification of the classical DoG model, that takes into consideration a disinhibitory contribution from the amacrine cells 12. This model is based on the existence of an extended surround beyond the classical receptive field. Such an extra-classical receptive field is reported both 
for LGN cells as well as the retinal ganglion cells [1314. The proposed Modified DoG model is:

$$
\begin{aligned}
\operatorname{MDOG}\left(\sigma_{1}, \sigma_{2}, \sigma_{3}\right)= & A_{1} \frac{1}{\sqrt{2 \pi} \sigma_{1}} \exp \left(-\frac{x^{2}}{2 \sigma_{1}{ }^{2}}\right)-A_{2} \frac{1}{\sqrt{2 \pi} \sigma_{2}} \exp \left(-\frac{x^{2}}{2 \sigma_{2}{ }^{2}}\right) \\
& +A_{3} \frac{1}{\sqrt{2 \pi} \sigma_{3}} \exp \left(-\frac{x^{2}}{2 \sigma_{3}{ }^{2}}\right)
\end{aligned}
$$

This simple computational model is capable of explaining the White effect. If we convolve Figure 3 with this model, we find that the convoluted image bears

(a)

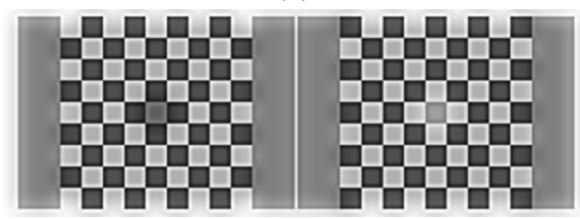

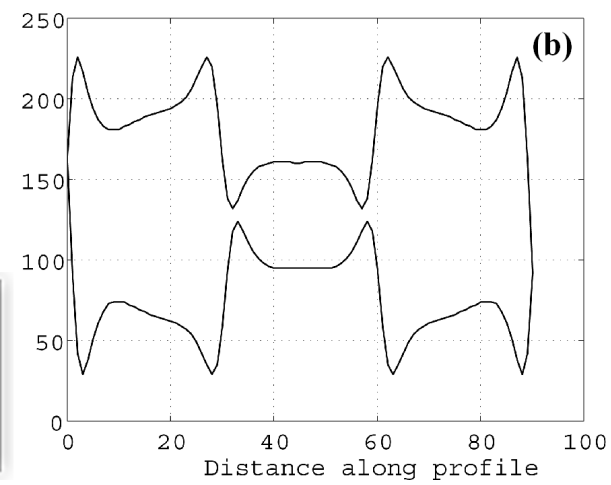

Fig. 6. (a) The Checkerboard stimulus when convoluted with MDoG. (b) Horizontal line profiles through the two test patches in (a). Here again the test patch in brighter neighbourhood has become brighter.

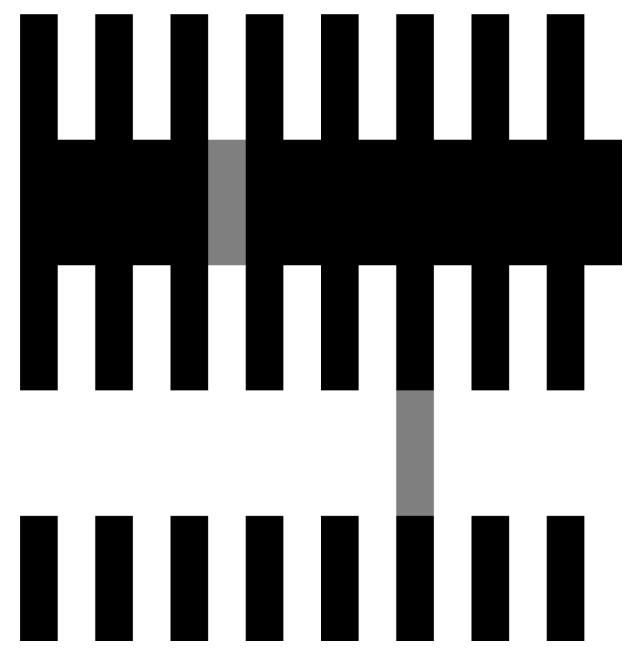

Fig. 7. The Howe stimulus 


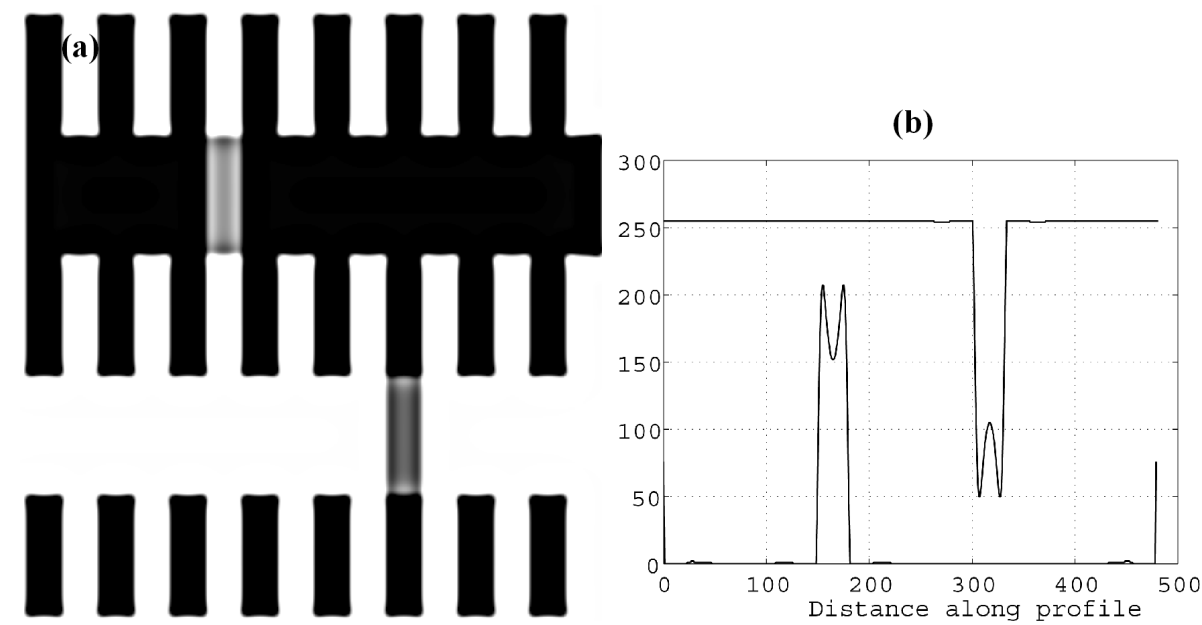

Fig. 8. (a) The Howe stimulus when convoluted with MDoG. (b) Horizontal line profiles through the two test patches in (a). Here again, unlike the White effect the test patch in brighter neighbourhood has become darker.

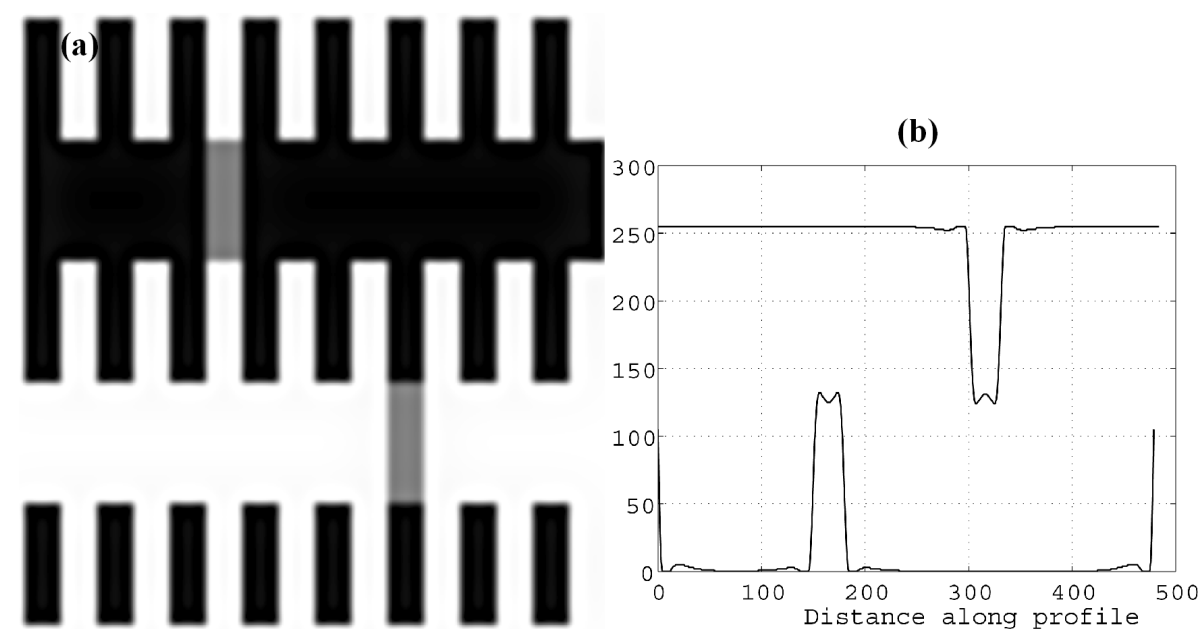

Fig. 9. (a) The Howe stimulus when convoluted with MDoG for the alternative settings. (b) Horizontal line profiles through the two test patches in (a). Here neighbourhood has no effect on the luminance of the test patches.

signature of the illusory perception in Figure 4 (a). This can again be verified by drawing horizontal profiles through the two test patches, with two neighbouring stripes on either side of each, as shown in Figure 4(b). A similar stimulus is the checkerboard illusion [6], shown in Figure 5. This is also inexplainable with the isotropic DoG model, since the test patch with darker neighbourhood on the left, appears less bright than the one with brighter neighbourhood on the right. This 
Gaussian Filtered Image
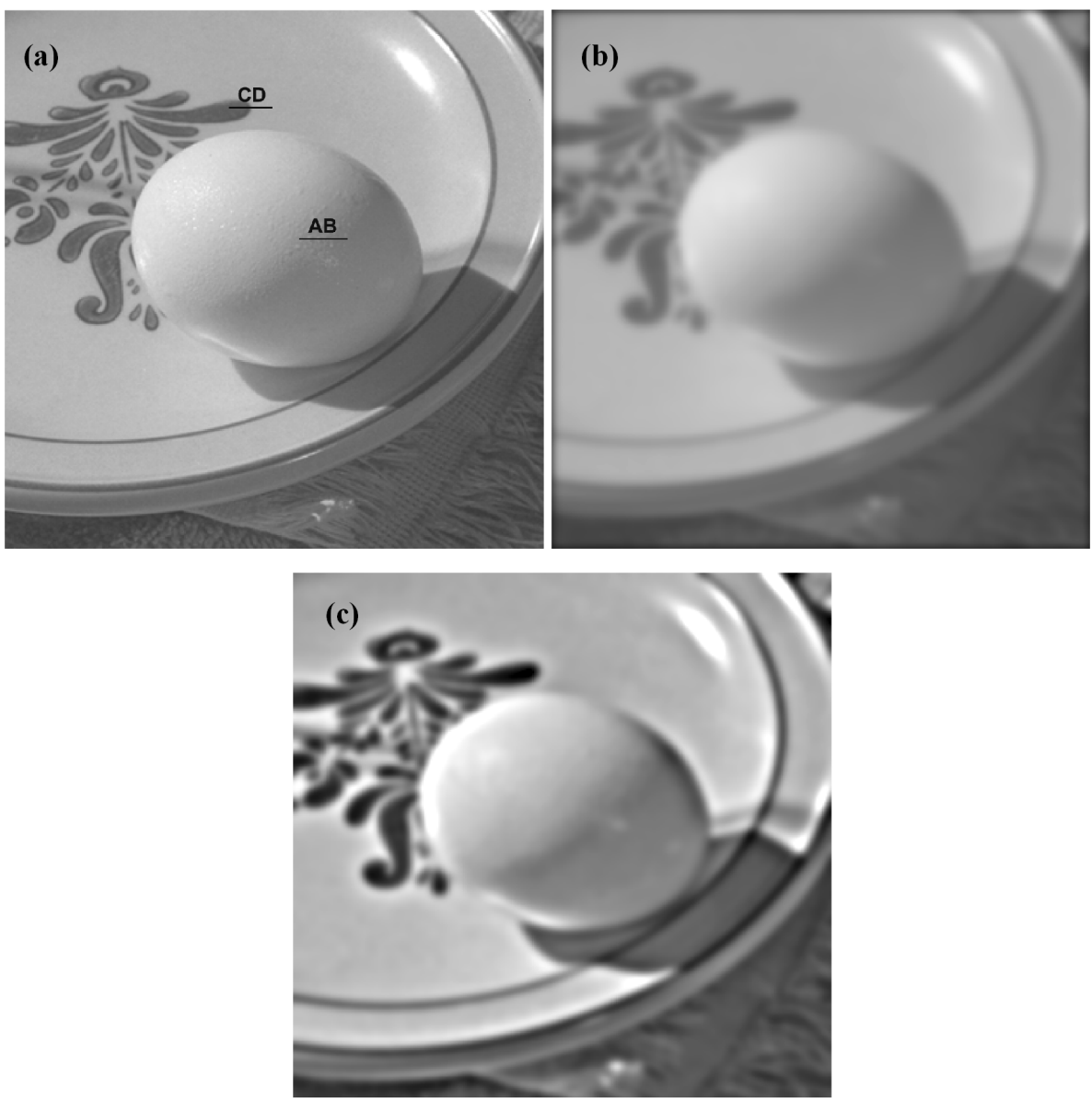

Fig. 10. (a) The benchmark image of egg-on-plate. (b) The resultant image when (a) is convoluted with a Gaussian function. (c) The resultant image when (a) is convoluted with the MDoG function.

illusion can also be explained with the help of the modified model for exactly the same parameter values as for explaining the White effect. This can be seen in Figure 6(a) and 6(b).

\subsection{The Gestalt Theories and Their Limitations}

The White effect and checkerboard illusions have led many investigators to abandon spatial filtering and receptive field based explanations, not only for these particular cases, but for brightness perception in general. Consequently, three explanations of the White effect that have been put forward are all certain rule based theories. For example Todorovic [15] put forward a T-junction rule and Anderson [16] also suggested a lightness rule. The computational model based 

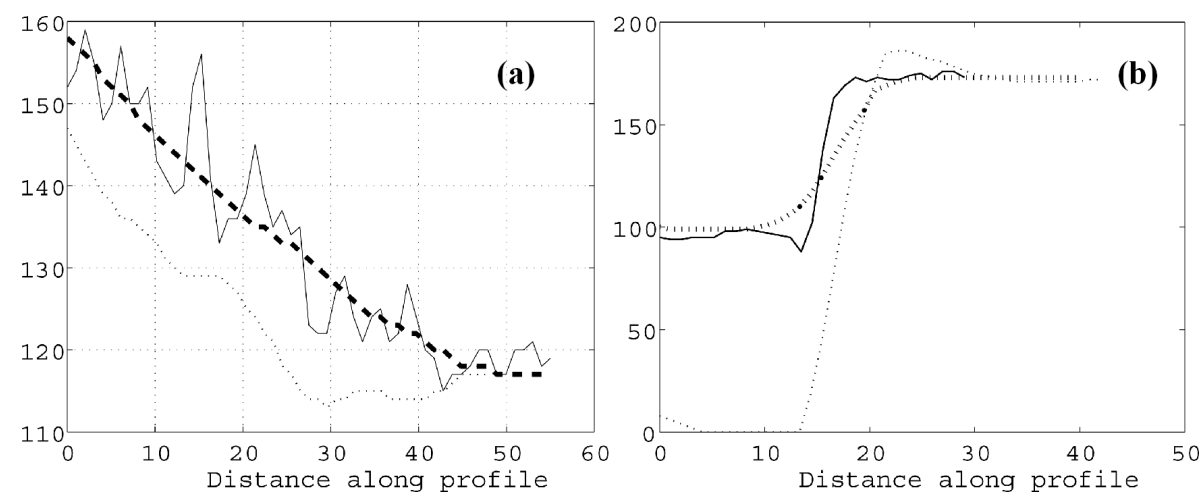

Fig. 11. (a) The intensity profile of AB marked in Figure 10(a) has been plotted with a solid line. The thicker dotted line represents the same line profile in Figure 10(b) and the thinner dotted line, that in Figure 10(c). Clearly, the MDoG function can behave as a smoothing function, like the Gaussian. (b) The intensity profile of CD marked in Figure 10(a) has been plotted with a solid line. The coarser dotted line represents the same line profile in Figure 10(b) and the finer dotted line, that in Figure 10(c). Clearly, the MDoG function can also behave as an edge enhancing function, like any derivative operator and unlike the Gaussian.

on higher level groupings that was forwarded by Ross and Pessoa [11] in its simplified form, also segments visual scenes by means of T-junctions. However, all the three explanations are incomplete if we consider the Howe effect, a novel variation of White's display [8] shown in Figure 7, where the T-junctions remain exactly the same compared to Figure 3 and yet the image perception changes. It was reported by Howe [8], that when this stimulus was observed by thirty-three naive observers, almost all of them reported either an opposite perception of brightness compared to White effect or no illusory perception at all. None of the three Gestalt explanations mentioned above, can explain these perceptions.

\section{Results with the Proposed Dynamic Model}

The modified DoG model proposed in the previous section has been found to be very effective in explaining many brightness-contrast illusions [9]. From this work, we find that the model is actually a dynamic one that operates at practically three different sets of amplitudes $\left(A_{1}, A_{2}\right.$ and $\left.A_{3}\right)$ and sampling intervals depending upon the illusory stimulus. If we apply the set of values used for explaining SBC by the proposed model [9], then convoluting Figure 7 with this model we achieve an explanation of the perception of those seventeen observers who reported an opposite perception of brightness compared to White effect. This has been shown in Figure 8 (a) and in its horizontal line profiles in Figure 8 (b).

If on the other hand, we apply the settings for explaining Mach band and Hermann grid illusion [9] when convoluting, we arrive at the explanation of 
Gaussian Filtered Image
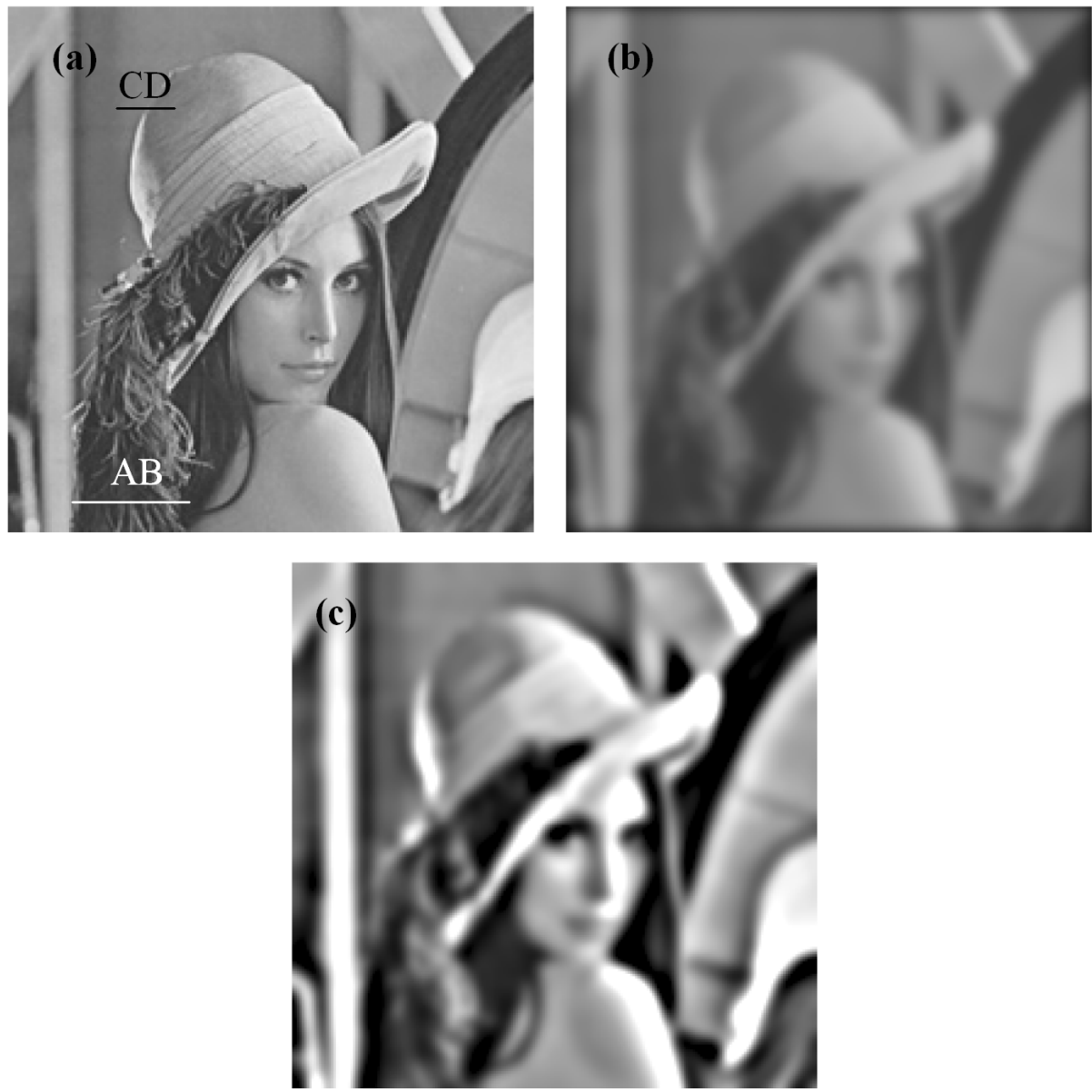

Fig. 12. (a) The benchmark image of Lena. (b) The resultant image when (a) is convoluted with a Gaussian function. (c) The resultant image when (a) is convoluted with the MDoG function.

those thirteen observers who reported equiluminance for both the patches. The convolution result and its corresponding line profiles are shown in Figure 9 (a) and $9(\mathrm{~b})$.

Finally, we apply the proposed filter on benchmark images and analyse the results. We find that the proposed filter behaves simultaneously as a smoothing function and an edge enhancer, in contrast to a normal Gaussian function that can only act as a smoothing function. This is clear from Figures 10 and 11 using the benchmark image egg-on-plate. Thus unlike the Laplacian of Gaussian function [17, which is an equivalent to the DoG, even without applying a derivative filter, we have achieved an enhancement of edges, together with smoothing 

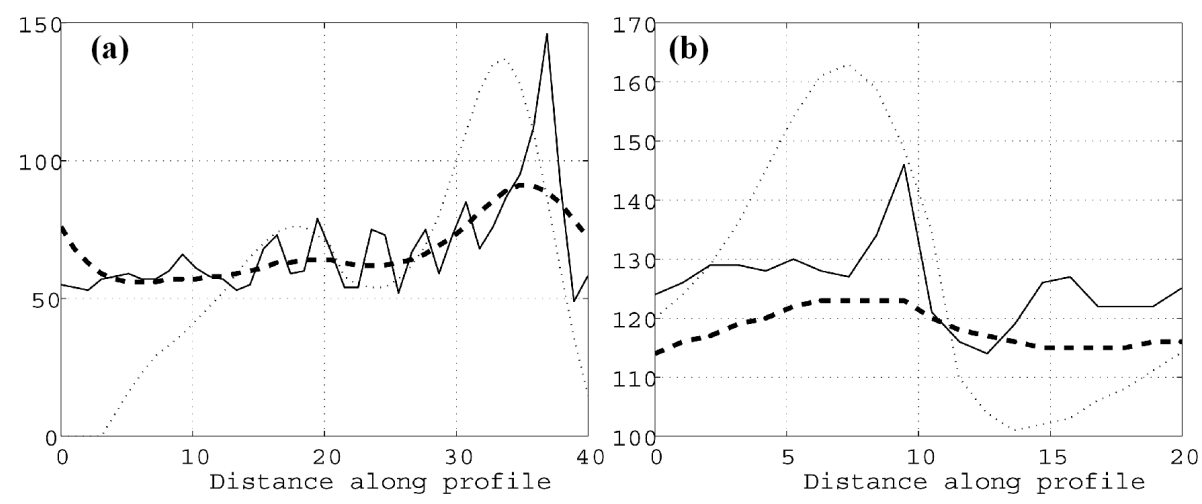

Fig. 13. (a) The intensity profile of $\mathrm{AB}$ marked in Figure 12(a) has been plotted with a solid line. The thicker dotted line represents the same line profile in Figure 12(b) and the thinner dotted line, that in Figure 12(c). Clearly, we find again that the MDoG function can behave as a smoothing function, like the Gaussian. (b) The intensity profile of CD marked in Figure 12(a) has been plotted with a solid line. The thicker dotted line represents the same line profile in Figure 12(b) and the thinner dotted line, that in Figure 12(c). So we find again that the MDoG function can also behave as an edge enhancing function, like any derivative operator and unlike the Gaussian.

using a linear combination of three Gaussian functions at different scales. For the benchmark image (Figure 10(a)) we have selected two horizontal line profiles $\mathrm{AB}$ and $\mathrm{CD}$ in two different regions of the image and compared the effects along these two profiles, after applying the Gaussian filter (Figure 10(b)) and the proposed filter (Figure 10(c)) respectively. The corresponding line profiles in Figure 11(a), show that like with the Gaussian, a smoothing has been achieved along the profile AB, with the proposed filter as well. On the other hand, the line profiles in Figure 11(b), show that unlike the Gaussian the proposed filter at the same time enhances the edge along the profile CD. Another benchmark image of Lena has similarly been processed and analyzed in Figures 12 and 13, yielding similar results.

\section{Conclusion}

Evidences in favour of a dynamic model of primary visual processing is presented here, based on a step-by-step information processing approach in contrast to the rule-based Gestalt approach. The model is able to explain not only the low-level brightness-contrast illusions, but also those, which were so far supposedly totally outside the purview of low-level vision. It is also capable of enhancing image edges without direct differentiation. Hence smoothing and edge enhancement may simultaneously be achieved in this new biologically inspired approach. 


\section{References}

1. Barlow, H. B.: Single units and sensation: a neuron doctrine for perceptual psychology? Perception 1 (1972) 371-394

2. Hubel D. H. and Wiesel T. N.: Receptive fields of single neurons in the cat's striate cortex. Journal of Physiology 148 (1959) 574-591

3. Hubel D. H. and Wiesel T. N.: Receptive fields, binocular interaction and functional architecture in the cat's visual cortex.Journal of Physiology 166 (1962) 106-154

4. Palmer, S. E.: Vision Science: Photons to Phenomenology, MIT Press, Cambridge, Massachusetts (1999)

5. White, M: The effect of the nature of the surround on the perceived lightness of gray bars within square-wave test gratings. Perception 10 (1981) 215-230

6. DeValois, R. L. and DeValois, K. K.: Spatial Vision, Oxford University Press, New York (1988)

7. Agostini, T. and Proffitt D. R.: Perceptual organization evokes simultaneous lightness contrast. Perception 22 (1993) 263-272

8. Howe, P. D. L.: A comment on the Anderson (1997), the Todorovic (1997), and the Ross and Pessoa (2000) explanation of White's effect. Perception 30 (2001) 1023-1026

9. Ghosh, K., Sarkar, S. and Bhaumik, K.: A possible explanation of the low-level brightness-contrast illusions in the light of an extended classical receptive field model of retinal ganglion cells. Biological Cybernetics 94 (2006) 89-96

10. Ghosh, K., Sarkar, S. and Bhaumik, K.: Proposing new methods in low-level vision from the Mach band illusion in retrospect. Pattern Recognition 39 (2006) 726-730

11. Ross, W. D. and Pessoa, L.: Lightness from contrast: A selective integration model. Perception and Psychophysics 62 (2000) 1160-1181

12. Ghosh, K., Sarkar, S. and Bhaumik, K.: A possible mechanism of zero-crossing detection using the concept of extended classical receptive field of retinal ganglion cells. Biological Cybernetics 93 (2005) 1-5

13. Sun, C., Chen, X., Huang, L. and Shou, T.: Orientation bias of the extraclassical re-ceptive field of the relay cells in the cat's dorsal lateral geniculate nucleus. Neuroscience 125 (2004) 495-505

14. Passaglia, L., Enroth-Cugell C. and Troy, J. B.: Effects of remote stimulation on the mean firing rate of cat retinal ganglion cells. Journal of Neuroscience 21 (2001) 5794-5803.

15. Todorovic, D.: Lightness and junctions. Perception 26 (1997) 379-395

16. Anderson, B. L.: A theory of illusory lightness and transparency in monocular and binocular images: the role of computer junctions. Perception 26 (1997) 419-453

17. Marr, D., Hildreth, E.: Theory of edge detection. Proceedings of Royal Society of London B 207 (1980) 187-217. 